\title{
Increased Resistance of SARS-CoV-2 Variants \\ B.1.351 and B.1.1.7 to Antibody Neutralization
}

David Ho ( $\nabla$ dh2994@cumc.columbia.edu )

Columbia University Medical Center https://orcid.org/0000-0003-1627-149X

\section{Pengfei Wang}

Aaron Diamond AIDS Research Center, Columbia University Irving Medical Center

Lihong Liu

Aaron Diamond AIDS Research Center, Columbia University Vagelos College of Physicians and

Surgeons, New York, NY, USA

Sho lketani

Columbia University

Yang Luo

Aaron Diamond AIDS Research Center, Columbia University Vagelos College of Physicians and

Surgeons, New York, NY, USA

\section{Yicheng Guo}

Zukerman Mind Brain Behavior Institute, Columbia University https://orcid.org/0000-0001-5357-660X

\section{Maple Wang}

Columbia University Medical Center

Jian Yu

Aaron Diamond AIDS Research Center, Columbia University Vagelos College of Physicians and

Surgeons, New York, NY, USA

\section{Baoshan Zhang}

Vaccine Research Center, NIAID, NIH

\section{Peter Kwong}

Vaccine Research Center, National Institute of Allergy and Infectious Diseases, National Institutes of Health

\section{Barney Graham}

$\mathrm{NIH} /$ VRC

John Mascola

National Institute of Allergy and Infectious Diseases

Jennifer Chang

Columbia University Medical Center

\section{Michael Yin}

Department of Biochemistry, Columbia University, New York, NY, USA.

\section{Magdalena Sobieszczyk}


Columbia University Medical Center

\section{Christos Kyratsous}

Regeneron Pharmaceuticals, Inc., https://orcid.org/0000-0002-2596-2906

\section{Lawrence Shapiro}

Columbia University https://orcid.org/0000-0001-9943-8819

\section{Zizhang Sheng}

Zukerman Mind Brain Behavior Institute, Columbia University

\section{Manoj Nair}

Aaron Diamond AIDS Research Center, Columbia University Vagelos College of Physicians and

Surgeons, New York, NY, USA https://orcid.org/0000-0002-5994-3957

\section{Yaoxing Huang}

Aaron Diamond AIDS Research Center, Columbia University Irving Medical Center https://orcid.org/0000-0001-6270-1644

\section{Biological Sciences - Article}

Keywords: SARS-CoV-2, variants, vaccine, antibody neutralization, resistance

Posted Date: January 29th, 2021

DOI: https://doi.org/10.21203/rs.3.rs-155394/v1

License: (c) (i) This work is licensed under a Creative Commons Attribution 4.0 International License. Read Full License

Version of Record: A version of this preprint was published at Nature on March 8th, 2021. See the published version at https://doi.org/10.1038/s41586-021-03398-2. 


\section{Increased Resistance of SARS-CoV-2 Variants B.1.351 and B.1.1.7 to Antibody Neutralization}

Pengfei Wang1,\#, Lihong Liu" ${ }^{1, \#}$, Sho Iketani ${ }^{1,2, \#, ~ Y a n g ~ L u o ~}{ }^{1}$, Yicheng Guo ${ }^{1}$, Maple Wang ${ }^{1}$, Jian Yu' ${ }^{1}$, Baoshan Zhang ${ }^{3}$, Peter D. Kwong ${ }^{3,4}$, Barney S. Graham ${ }^{3}$, John R. Mascola ${ }^{3}$, Jennifer Y. Chang ${ }^{1,5}$, Michael T. Yin ${ }^{1,5}$, Magdalena Sobieszczyk ${ }^{1,5}$, Christos A. Kyratsous $^{6}$, Lawrence Shapiro ${ }^{1,4,7}$, Zizhang Sheng ${ }^{1}$, Manoj S. Nair ${ }^{1}$, Yaoxing Huang ${ }^{1}$, \& David D. Ho ${ }^{1,2,5, *}$

${ }^{1}$ Aaron Diamond AIDS Research Center, Columbia University Vagelos College of Physicians and Surgeons, New York, NY, USA. '2Department of Microbiology and Immunology, Columbia University Irving Medical Center, New York, NY, USA. ${ }^{3}$ Vaccine Research Center, National Institutes of Health, Bethesda, MD, USA. ${ }^{4}$ Department of Biochemistry, Columbia University, New York, NY, USA. ${ }^{5}$ Division of Infectious Diseases, Department of Internal Medicine, Columbia University Vagelos College of Physicians and Surgeons, New York, NY, USA. ${ }^{6}$ Regeneron Pharmaceuticals, Inc., Tarrytown, NY. USA. ${ }^{7}$ Zuckerman Mind Brain Behavior Institute, Columbia University, New York, NY, USA. \#These authors contributed equally: Pengfei Wang, Lihong Liu, and Sho Iketani. "Address correspondence to dh2994@cumc.columbia.edu 
The Covid-19 pandemic has ravaged the globe, and its causative agent, SARS-CoV2, continues to rage. Prospects of ending this pandemic rest on the development of effective interventions. Two monoclonal antibody (mAb) therapeutics have received emergency use authorization ${ }^{1,2}$, and more are in the pipeline ${ }^{3-6}$. Furthermore, multiple vaccine constructs have shown promise ${ }^{7}$, including two with $\sim 95 \%$ protective efficacy against Covid-198,9. However, these interventions were directed toward the initial SARS-CoV-2 that emerged in 2019. Considerable viral evolution has occurred since, including variants with a D614G mutation ${ }^{10}$ that have become dominant. Viruses with this mutation alone do not appear to be antigenically distinct, however ${ }^{11}$. Recent emergence of new SARS-CoV-2 variants B.1.1.7 in the UK ${ }^{12}$ and B.1.351 in South Africa ${ }^{13}$ is of concern because of their purported ease of transmission and extensive mutations in the spike protein. We now report that B.1.1.7 is refractory to neutralization by most mAbs to the $\mathrm{N}$ terminal domain (NTD) of spike and relatively resistant to a number of mAbs to the receptor-binding domain (RBD). It is modestly more resistant to convalescent plasma ( $\sim 3$ fold) and vaccinee sera ( $\sim 2$ fold). Findings on B.1.351 are more worrisome in that this variant is not only refractory to neutralization by most NTD mAbs but also by multiple potent mAbs to the receptor-binding motif on RBD, largely due to an E484K mutation. Moreover, B.1.351 is markedly more resistant to neutralization by convalescent plasma ( 11-33 fold) and vaccinee sera ( 6.5-8.6 fold). B.1.351 and emergent variants ${ }^{14,15}$ with similar spike mutations present new challenges for mAb therapy and threaten the protective efficacy of current vaccines. 
SARS-CoV-2 B.1.1.7, also known as 501Y.V1 in the GR clade (Fig. 1a), emerged in September 2020 in South East England and rapidly became the dominant variant in the UK, possibly due to its enhanced transmissibility ${ }^{12}$. This strain has now spread to over 50 countries. B.1.1.7 contains 8 spike mutations in addition to D614G, including two deletions (69-70del \& 144del) in NTD, one mutation (N501Y) in RBD, and one mutation $(\mathrm{P} 681 \mathrm{H})$ near the furin cleavage site (Fig. 1b). SARS-CoV-2 B.1.351, also known as 501Y.V2 in the GH clade (Fig. 1a), emerged in late 2020 in Eastern Cape, South Africa $(\mathrm{SA})^{13}$. This variant has since become dominant locally, raising the specter that it too has enhanced transmissibility. B.1.351 contains 9 spike mutations in addition to D614G, including a cluster of mutations (e.g., 242-244del \& R246I) in NTD, three mutations (K417N, E484K, \& N501Y) in RBD, and one mutation (A701V) near the furin cleavage site (Fig. 1b). There is a growing concern that these new variants could impair the efficacy of current mAb therapies or vaccines, because many of the mutations reside in the antigenic supersite in NTD ${ }^{16,17}$ or in the ACE2-binding site (also known as the receptorbinding motif-RBM) that is a major target of potent virus-neutralizing antibodies. We therefore addressed this concern by creating VSV-based SARS-CoV-2 pseudoviruses that contain each of the individual mutations as well as one with all 8 mutations of the B.1.1.7 variant $(\mathrm{UK} \Delta 8)$ and another with all 9 mutations of the B.1.351 variant (SA $\Delta 9)$. A total of 18 mutant pseudoviruses were made as previously described ${ }^{18,19}$, and each was found to have a robust titer (Extended Data Fig. 1) adequate to measure its susceptibility to neutralization by $30 \mathrm{mAbs}, 20$ convalescent plasma, and 22 vaccinee sera. 


\section{Monoclonal antibodies}

We first assayed the neutralizing activity of 12 RBD mAbs against UK $\Delta 8, \mathrm{SA} \Delta 9$, and WT (D614G) pseudoviruses in Vero E6 cells as previously described ${ }^{18,19}$. Three mAbs target the "inner side", four target RBM, and five target the "outer side". The footprints of these mAbs on RBD are shown in Fig. 2a, and their neutralization profiles are shown in Fig. 2b. For neutralization of UK $\Delta 8$, only the activities of $910-30^{20}$ and $S 309^{4}$ are impaired, albeit modestly. For neutralization of SA $\Delta$, however, the activities of $910-30,2-15^{18}$, LYCoV555 (bamlanivimab) ${ }^{1,21}$, C12122, and REGN10933 (casirivimab) ${ }^{2}$ are completely or markedly abolished. The four mAbs that target RBM are among the most potent SARSCoV-2-neutralizing antibodies in clinical use or development. Note that mAbs directed to lower aspects of the "inner side" $\left(2-36^{18} \&\right.$ COVA1-16 $\left.{ }^{23,24}\right)$ or to the "outer side" retain their activities against SA $\Delta$, including 2-7 ${ }^{18}$, REGN10987 (imdevimab) ${ }^{2}, \mathrm{C} 135^{22}$, and S309 that are in clinical use or development. The results on the neutralization of UK $\Delta 8$ and SA $\triangle 9$ by these $12 \mathrm{mAbs}$ are summarized in Fig. $2 \mathrm{c}$ as fold changes in IC50 neutralization titers relative to the WT. To understand the specific spike mutations responsible for the observed changes, we also tested the same panel of mAbs against pseudoviruses containing only a single mutation found in B.1.1.7 or B.1.351. The results are displayed, among others, in Extended Data Fig. 2 and summarized in Fig. 2c. Against UK $\Delta 8$, the decreased activity of $910-30$ is mediated by N501Y, whereas the slightly impaired activity of $S 309$ is unexplained. Against $S A \Delta 9$, the complete loss of activity of 2-15, LY-CoV555, and C121 is mediated by E484K; the complete loss for $910-30$ is mediated by K417N; and the marked reduction for REGN10933 is mediated by K417N 
and E484K. A structural explanation on how E484K disrupts the binding of 2-15, LYCoV555, and REGN10933 is presented in Extended Data Fig. 3a.

We also assessed the neutralizing activity of six NTD mAbs against UK $\Delta 8, \mathrm{SA} \Delta 9$, and WT pseudoviruses. Both UK $\Delta 8$ and $\mathrm{SA} \Delta 9$ are profoundly resistant to neutralization by our antibodies $5-24,4-8,2-17$, and $4-19^{18}$, as well as by $4 A 8^{25}$ (Fig. 2 d). Note that $5-24$, 4A8, and 4-8 are known to target the antigenic supersite in NTD ${ }^{16}$ (Insert in Fig. 2d). The activity of $5-7^{18}$ remains intact, however. To understand the specific mutations responsible for the observed changes, we then tested these mAbs against pseudoviruses containing only a single mutation found in B.1.1.7 or B.1.351 (Extended Data Fig. 2). The results are summarized in Fig. $2 \mathrm{c}$ as fold change relative to the WT. It is evident that the resistance of UK $\Delta 8$ to most NTD mAbs is largely conferred by $144 \mathrm{del}$, whereas the resistance of SA $\Delta 9$ is largely conferred by $242-244$ del and/or R246I. Amino-acid residues 144, 242-244, and 246 all fall within the NTD supersite ${ }^{16,17}$ (Insert in Fig. 2d; details in Extended Data Fig. 3b). The obvious exception is 5-7, whose neutralizing activity is actually enhanced. Needless to say, a detailed structural understanding of how 5-7 binds NTD will be important.

We next tested the neutralizing activity of 12 additional RBD mAbs, including ones from our own collection $(1-20,4-20,2-4,2-43,2-30, \& 2-38)^{18}$ as well as CB65 ${ }^{5}$ COV2-2196 \& COV2-21306, Brii-196 \& Brii-198³, and REGN10985. The results against UK $\Delta 8, \mathrm{SA} \Delta 9$, and WT are highlighted in Extended Data Fig. 4a, and the detailed findings against the single-mutation pseudoviruses are shown in Extended Data Fig. 2. The fold changes in 
neutralization IC50 titers relative to the WT are tabulated in Extended Data Fig. 4b. Herein we only comment on results for mAbs in clinical development. The activity of CB6 is slightly impaired against UK $\Delta 8$, likely due to N501Y and/or S982A, but it is rendered inactive against SA $\triangle 9$ because of K417N. Brii-196 and COV2-2130 are essentially unaffected by the new variants; the activities of Brii-198 and COV2-2196 are slightly diminished against $\mathrm{SA} \Delta 9$ but not against $\mathrm{UK} \Delta 8$.

Lastly, we examined, in a single experiment, the neutralizing activity of mAb therapies in clinical use or under clinical investigation against UK $\Delta 8, \mathrm{SA} \Delta 9$, and D614G pseudoviruses. The results for single mAbs LY-CoV555 and S309, as well as for combination regimens REGN10933+REGN10987, LY-CoV555+CB6, Brii-196+Brii-198, and COV2-2196+COV2-2130, are shown in Extended Data Fig. 5 and summarized in Fig. 2e. Note that LY-CoV555, alone or in combination with CB6, is no longer able to neutralize SA $\Delta$ 9. While REGN10933+REGN10987 and COV2-2196+COV2-2130 are seemingly unaffected, each of these potent combinations has a component that has lost some neutralizing activity (Fig. 2c \& Extended Data Fig. 4b). Although S309 and the Brii196+Brii-198 combination are not significantly impaired, their potencies are noticeably lower (Fig. 2e). These findings suggest that antibody treatment of this virus might need to be modified in localities where B.1.351 and related variants ${ }^{14,15}$ are prevalent, and highlight the importance of combination antibody therapy to address the expanding antigenic diversity of SARS-CoV-2.

\section{Convalescent plasma}


We obtained convalescent plasma from 20 patients more than one month after documented SARS-CoV-2 infection in the Spring of 2020. Ten had severe disease and 10 had non-severe disease, as previously defined ${ }^{19}$. Their ages ranged from 34 to 79 , with a mean of 54 . Six were female, and 14 were male.

Each plasma sample was then assayed for neutralization against UK $\Delta 8, \mathrm{SA} \Delta 9$, and WT pseudoviruses. Fig. 3a shows that most plasma samples lost $>2$-fold neutralizing activity against the new variants relative to the WT. The loss in potency is more frequent against $\mathrm{SA} \Delta 9$ (16 of 20 ) than against $\mathrm{UK} \Delta 8$ (11 of 20 ). Only plasma from $\mathrm{P} 7, \mathrm{P} 10, \mathrm{P} 18$, and P20 retain neutralizing activities identical or similar to those against the WT. These results are summarized as fold change in plasma neutralization IC50 titers in Fig. 3b. Furthermore, the magnitude of the drop in plasma neutralization is better seen in Fig. 3c, with the overall mean loss of activity being modest against UK $\Delta 8$ (2.7 to 3.8 fold), but more substantial against $\mathrm{SA} \Delta 9$ (11.0 to 33.1 fold).

Every plasma sample was also tested against each single-mutation pseudovirus, and those findings are shown in Extended Data Fig. 6 and summarized in Fig. 3b. Unlike the data for mAbs (Fig. 2c), no single mutation could predictably account for the loss of plasma neutralizing activity against $\mathrm{UK} \Delta 8$, indicating that the mutations in this variant do not perturb an immunodominant epitope on the spike that is shared by many infected persons. S982A seems to have a discernible negative impact on the plasma neutralizing activity of 9 samples (Fig. 3b), perhaps due to its interaction with the bottom of RBD (Extended Data Fig. 3c). On the other hand, the loss of plasma neutralizing activity 
against $\mathrm{SA} \Delta 9$ could be largely attributed to E484K (Fig. 3b), suggesting that this RBM mutation to be situated in an immunodominant epitope for most infected persons. It is also interesting to note that cases such as $\mathrm{P} 7$ and $\mathrm{P} 10$ have neutralizing antibodies that are essentially unperturbed by the multitude of spike mutations found in these two new variants (Fig. 3b). A detailed analysis of their antibody repertoire against the viral spike could be informative.

\section{Vaccinee Sera}

Sera were obtained from 12 participants of a Phase 1 clinical trial of Moderna SARS-Co-

2 mRNA-1273 Vaccine ${ }^{8}$ conducted at the $\mathrm{NIH}$. These volunteers received two immunizations with the vaccine $(100 \mu \mathrm{g})$ on days 0 and 28 , and blood was collected on day 43. Additional vaccinee sera were obtained at Columbia University Irving Medical Center from 10 health care workers who received the Pfizer BNT162b2 Covid-19 Vaccine $^{9}$ at the clinical dose on days 0 and 21 . Blood was collected on day 28 or later.

Each vaccinee serum sample was assayed for neutralization against UK $\Delta 8, \operatorname{SA} \Delta 9$, and WT pseudoviruses. Fig. 4a shows only a minority of sera to have lost $>2$-fold neutralizing activity against $\mathrm{UK} \Delta 8$, whereas every sample lost activity against $\mathrm{SA} \Delta 9$, ranging from slight to substantial. These results are quantified and tabulated as fold change in neutralization IC50 titers in Fig. 4b, and the extent of the decline in neutralization activity is more evident in Fig. 4c. Overall, the mean loss of neutralizing activity against UK $\Delta 8$ appears to be small (1.8 fold, Moderna; 2.0 fold, Pfizer), but quite significant against SA $\Delta 9$ (8.6 fold, Moderna; 6.5 fold, Pfizer). 
Every vaccinee serum was also tested against each single-mutation pseudovirus, and the results are presented in Extended Data Fig. 7 and summarized in Fig. 4b. As was the case for convalescent plasma (Fig. 3b), no single mutation could predictably account for the small loss of serum neutralizing activity against UK $\Delta 8$. Again, S982A seems to have a minor negative impact on the plasma neutralizing activity of every serum sample (Fig. 4b), possibly due to distal effects on the RBD (Extended Data Fig. 3c). The loss of neutralizing activity against $\mathrm{SA} \Delta 9$ in vaccinee sera could be principally attributed to E484K (Fig. 4b), indicating that this RBM mutation to be situated in an immunodominant epitope recognized by all vaccinees studied. Our findings do not reveal any significant differences between the two different vaccines.

\section{Discussion}

Both SARS-CoV-2 variants B.1.1.7 and B.1.351 are raising concerns not only because of their increase transmissibility but also because of their extensive mutations in spike that could lead to antigenic changes detrimental to $\mathrm{mAb}$ therapies and vaccine protection. It is of equal concern that another variant, B.1.1.28 or $501 Y . \mathrm{V} 3$, is increasing rapidly in Brazil and spreading far beyond ${ }^{14,15}$. B.1.1.28 contains three mutations (K417T, E484K, and N501Y) at the same RBD residues as B.1.351. Much of our findings on SA $\Delta 9$ would likely be similar for this emergent variant. N501Y is shared among viruses in these three lineages; while this mutation may confer enhanced binding to $\mathrm{ACE} 2^{26}$, its antigenic impact is limited to a few mAbs (Fig. 2c \& Extended Data Fig. 4b), with no pronounced effects on the neutralizing activity of convalescent plasma or vaccinee sera (Figs. $3 b \& 4 b$ ). 
Our findings have relevance to the use of mAb to treat or prevent SARS-CoV-2. Both UK $\Delta 8$ and $\mathrm{SA} \Delta 9$ are resistant to neutralization by mAbs directed to the NTD supersite (Figs. 2c, 2d, \& Extended Data Fig. 3b). More importantly, SA $\Delta 9$ is resistant to a major group of potent mAbs that target the RBM, including two authorized for emergency use (Fig. 2c). LY-CoV555 is inactive against $\mathrm{SA} \Delta 9$, and the $\sim 60$-fold loss in potency of REGN10933 renders the combination of REGN10933+REGN10987 to be effectively monotherapy. Several other mAbs in development are similarly impaired (Figs. 2c, 2e, \& Extended Data Fig. 4b) against this variant. Decisions on the use of these mAbs will depend heavily on the local prevalence of B.1.351 or B.1.1.28, thus highlighting the importance of viral genomic surveillance worldwide and proactive development of nextgeneration antibody therapeutics.

Convalescent plasma from patients infected with SARS-CoV-2 from early in the pandemic show slightly decreased neutralizing activity against $\mathrm{UK} \Delta 8$, but the diminution against $\mathrm{SA} \Delta 9$ is remarkable (Figs. $3 \mathrm{~b} \& 3 \mathrm{c}$ ). This relative resistance is largely due to E484K, a mutation shared by B.1.351 and B.1.1.28 $8^{13-15}$. Again, in areas where such viruses are common, one would have heightened concerns about re-infection, which has already been well documented even in the absence of antigenic changes ${ }^{27,28}$.

As for the ramifications of our findings for the protective efficacy of current SARS-CoV-2 vaccines, the $\sim 2$-fold loss of neutralizing activity of vaccinee sera against UK $\Delta 8$ is unlikely to have an adverse impact due to the large "cushion" of residual neutralizing antibody titer 
(Fig. $4 \mathrm{c}$ ). On the other hand, the loss of $\sim 6.5-8.6$ fold in activity against SA $\Delta 9$ is more worrisome, although the clinical implication for vaccine efficacy remains to be determined. The results from ongoing trials in South Africa using these or similar vaccine constructs should be informative.

The recent emergence of B.1.1.7, B.1.351, and B.1.1.28 is a clear demonstration of SARS-CoV-2 antigenic drift. This conclusion is supported by data presented herein, illustrating how so many of these spike changes conferred resistance from antibody neutralization. Mutationally, this virus is traveling in a direction that could ultimately lead to escape from our current therapeutic and prophylactic interventions directed to the viral spike. If the rampant spread of the virus continues and more critical mutations accumulate, then we may be condemned to chasing after the evolving SARS-CoV-2 continually, as we have long done for influenza virus. Such considerations require that we stop virus transmission as quickly as is feasible, by redoubling our mitigation measures and by expediting vaccine rollout. 


\section{References}

1 Chen, P. et al. SARS-CoV-2 Neutralizing Antibody LY-CoV555 in Outpatients with Covid-19. N Engl J Med 384, 229-237 (2021).

2 Hansen, J. et al. Studies in humanized mice and convalescent humans yield a SARS-CoV-2 antibody cocktail. Science 369, 1010-1014 (2020).

$3 \mathrm{Ju}, \mathrm{B}$. et al. Human neutralizing antibodies elicited by SARS-CoV-2 infection. Nature 584, 115-119 (2020).

4 Pinto, D. et al. Cross-neutralization of SARS-CoV-2 by a human monoclonal SARS-CoV antibody. Nature 583, 290-295 (2020).

5 Shi, R. et al. A human neutralizing antibody targets the receptor-binding site of SARS-CoV2. Nature 584, 120-124 (2020).

6 Zost, S. J. et al. Potently neutralizing and protective human antibodies against SARS-CoV2. Nature 584, 443-449 (2020).

$7 \quad$ Krammer, F. SARS-CoV-2 vaccines in development. Nature 586, 516-527 (2020).

8 Anderson, E. J. et al. Safety and Immunogenicity of SARS-CoV-2 mRNA-1273 Vaccine in Older Adults. N Engl J Med 383, 2427-2438 (2020).

9 Polack, F. P. et al. Safety and Efficacy of the BNT162b2 mRNA Covid-19 Vaccine. N Engl J Med 383, 2603-2615 (2020).

10 Korber, B. et al. Tracking Changes in SARS-CoV-2 Spike: Evidence that D614G Increases Infectivity of the COVID-19 Virus. Cell 182, 812-827 e819 (2020).

11 Hou, Y. J. et al. SARS-CoV-2 D614G variant exhibits efficient replication ex vivo and transmission in vivo. Science 370, 1464-1468 (2020).

12 Rambaut $A$, et al. Preliminary genomic characterisation of an emergent SARSCoV-2 lineage in the UK defined by a novel set of spike mutations. https://virological.org/t/preliminarygenomic-characterisation-of-an-emergent-sars-cov2-lineage-in-the-uk-defined-by-a-novel-set-ofspike-mutations/563. (Published 2020).

13 Tegally, H. et al. Emergence and rapid spread of a new severe acute respiratory syndromerelated coronavirus 2 (SARS-CoV-2) lineage with multiple spike mutations in South Africa. medRxiv, 2020.2012.2021.20248640 (2020).

14 Faria NR, et al. Genomic characterisation of an emergent SARS-CoV-2 lineage in Manaus: preliminary findings. https://virological.org/t/genomic-characterisation-of-an-emergentsars-cov-2-lineage-in-manaus-preliminary-findings/586. (Published 2021).

15 Naveca F, et al. Phylogenetic relationship of SARS-CoV-2 sequences from Amazonas with emerging Brazilian variants harboring mutations E484K and N501Y in the Spike protein. https://virological.org/t/phylogenetic-relationship-of-sars-cov-2-sequences-fromamazonas-with-emerging-brazilian-variants-harboring-mutations-e484k-and-n501y-inthe-spike-protein/585. (Published 2021).

16 Cerutti, G. et al. Potent SARS-CoV-2 Neutralizing Antibodies Directed Against Spike NTerminal Domain Target a Single Supersite. bioRxiv, 2021.2001.2010.426120 (2021).

17 McCallum, M. et al. N-terminal domain antigenic mapping reveals a site of vulnerability for SARS-CoV-2. bioRxiv, 2021.2001.2014.426475 (2021).

18 Liu, L. et al. Potent neutralizing antibodies against multiple epitopes on SARS-CoV-2 spike. Nature 584, 450-456 (2020). 
19 Wang, P. et al. SARS-CoV-2 neutralizing antibody responses are more robust in patients with severe disease. Emerg Microbes Infect 9, 2091-2093 (2020).

20 Banach, B. B. et al. Paired heavy and light chain signatures contribute to potent SARSCoV-2 neutralization in public antibody responses. bioRxiv, 2020.2012.2031.424987 (2021).

21 Jones, B. E. et al. LY-CoV555, a rapidly isolated potent neutralizing antibody, provides protection in a non-human primate model of SARS-CoV-2 infection. bioRxiv (2020).

22 Robbiani, D. F. et al. Convergent antibody responses to SARS-CoV-2 in convalescent individuals. Nature 584, 437-442 (2020).

23 Brouwer, P. J. M. et al. Potent neutralizing antibodies from COVID-19 patients define multiple targets of vulnerability. Science 369, 643-+ (2020).

24 Liu, H. et al. Cross-Neutralization of a SARS-CoV-2 Antibody to a Functionally Conserved Site Is Mediated by Avidity. Immunity 53, 1272-1280 e1275 (2020).

25 Chi, X. et al. A neutralizing human antibody binds to the N-terminal domain of the Spike protein of SARS-CoV-2. Science 369, 650-655 (2020).

26 Starr, T. N. et al. Deep Mutational Scanning of SARS-CoV-2 Receptor Binding Domain Reveals Constraints on Folding and ACE2 Binding. Cell 182, 1295-1310 e1220 (2020).

27 Tillett, R. L. et al. Genomic evidence for reinfection with SARS-CoV-2: a case study. Lancet Infect Dis 21, 52-58 (2021).

28 To, K. K. et al. COVID-19 re-infection by a phylogenetically distinct SARS-coronavirus-2 strain confirmed by whole genome sequencing. Clin Infect Dis (2020). 


\section{Figure legends}

Fig. 1 | Emerging SARS-CoV-2 variants identified in the UK and SA. a, Phylogenetic tree of SARS-CoV-2 variants, with B.1.351 and B.1.1.7 highlighted. b, Mutations in the viral spike identified in B.1.351 (SA) and B.1.1.7 (UK) in addition to D614G.

Fig. 2 | Susceptibility of UK $\triangle 8$ and $S A \Delta 9$ pseudoviruses to neutralization by mAbs. a, Footprints of neutralizing mAbs on the RBD. Left panel, top view of SARS-COV-2 spike with one RBD in the "up" conformation (pdb: 6zgg). RBD and NTD are colored green and peach, respectively. The positions of 'inner' and 'outer' sides are indicated on the "up" RBD with the ACE2-binding site colored yellow. The three panels to the right show the antibody footprints on RBD. b, Neutralization of UK $\Delta 8, \mathrm{SA} \Delta 9$, and WT pseudoviruses by select RBD mAbs. c, Fold-change in IC50 of neutralizing mAbs against UK $\Delta 8$ and SA $\Delta 9$, as well as single-mutation pseudoviruses, relative to WT. MPI $\downarrow$ denotes that maximum percent inhibition is substantially reduced, confounding IC50 calculations. d, Neutralization of UK $\Delta 8, \operatorname{SA} \Delta 9$, and WT pseudoviruses by NTD-directed mAbs, the footprints of which are delineated by the color tracings in the insert. e, Changes in neutralization IC50 of authorized or investigational therapeutic mAbs against UK $\Delta 8$ and $\mathrm{SA} \triangle 9$. Data in $\mathbf{b}$ and $\mathbf{d}$ are mean $\pm \mathrm{SEM}$ of technical triplicates, and represent one of two independent experiments.

Fig. 3 | UK $\triangle 8$ and SA $\triangle 9$ pseudoviruses are more resistant to neutralization by convalescent plasma from patients. a, Neutralization results for 20 convalescent plasma samples (P1-P20) against UK $\Delta 8, \mathrm{SA} \Delta 9$, and WT. Data represent mean $\pm \mathrm{SEM}$ of 
technical triplicates. The panels are arranged by IC50 values against the WT, from low to high. b, Fold change in neutralization IC50 of UK $\Delta 8$ and SA $\Delta 9$, as well as singlemutation pseudoviruses, relative to the WT presented as a heatmap with darker colors implying greater change. c, Change in reciprocal plasma neutralization IC50 values of convalescent plasma from severe and non-severe patients against UK $\Delta 8$ and $\mathrm{SA} \Delta 9$, relative to the WT. Mean fold changes in IC50 values relative to the WT are written above the $p$ values. Statistical analysis was performed using a two-tailed paired $t$ test.

Fig. 4 | UK $\Delta 8$ and SA $\Delta 9$ pseudoviruses are more resistant to neutralization by vaccinee sera. a, Neutralization profiles for 22 serum samples obtained from persons who received SARS-CoV-2 vaccine made by Moderna (V1-V12) or Pfizer (V13-V22) against $\mathrm{UK} \Delta 8, \mathrm{SA} \Delta 9$, and WT pseudoviruses. The panels are arranged by IC50 values against the WT, from low to high for each set of vaccinees. Data are mean \pm SEM of technical triplicates, and represent one of two independent experiments. b, Fold change in serum neutralization $\mathrm{IC} 50$ of $\mathrm{UK} \Delta 8$ and $\mathrm{SA} \Delta 9$, as well as single-mutation pseudoviruses, relative to the WT presented as a heatmap with darker colors implying greater change. c, Change in reciprocal serum IC50 values for Moderna and Pfizer vaccinees against UK $\Delta 8$ and $\mathrm{SA} \Delta 9$, relative to the WT. Mean fold change in IC50 relative to the WT is written above the $p$ values. Statistical analysis was performed using a twotailed paired $t$ test. 
Fig. 1

a

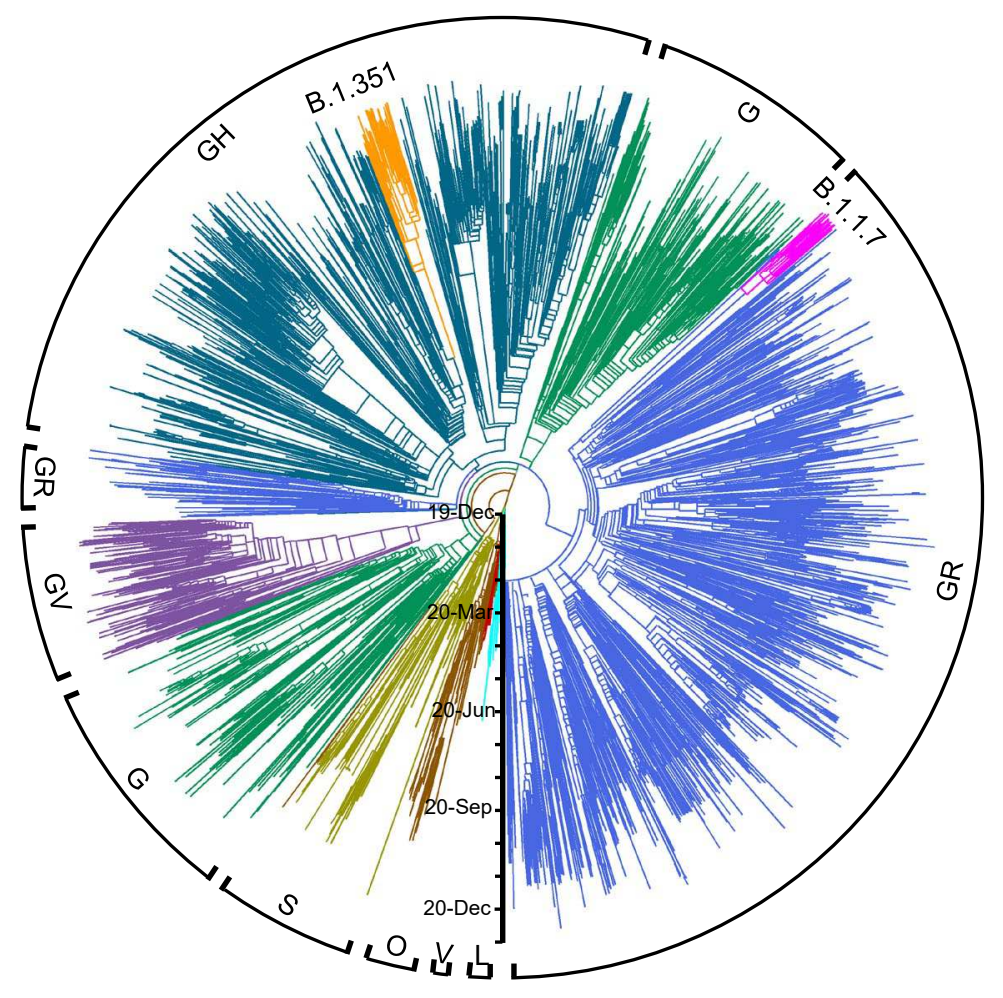

b

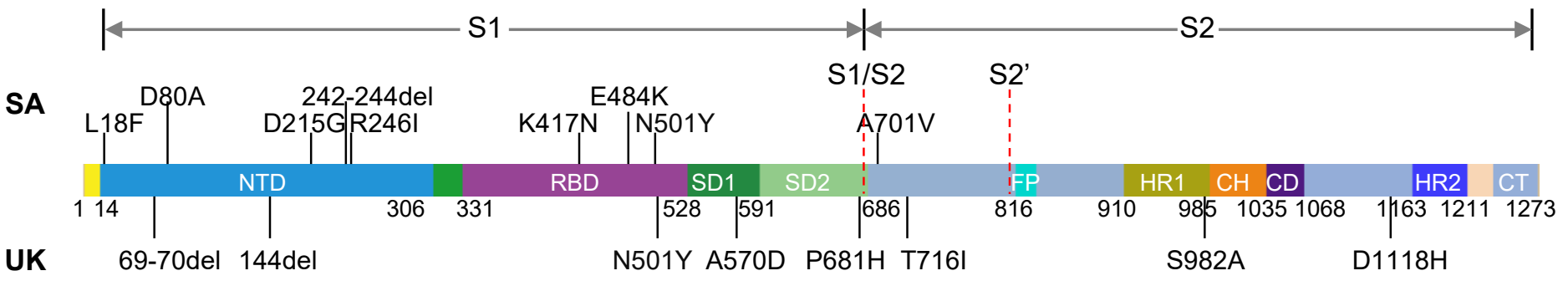


Fig. 2

a

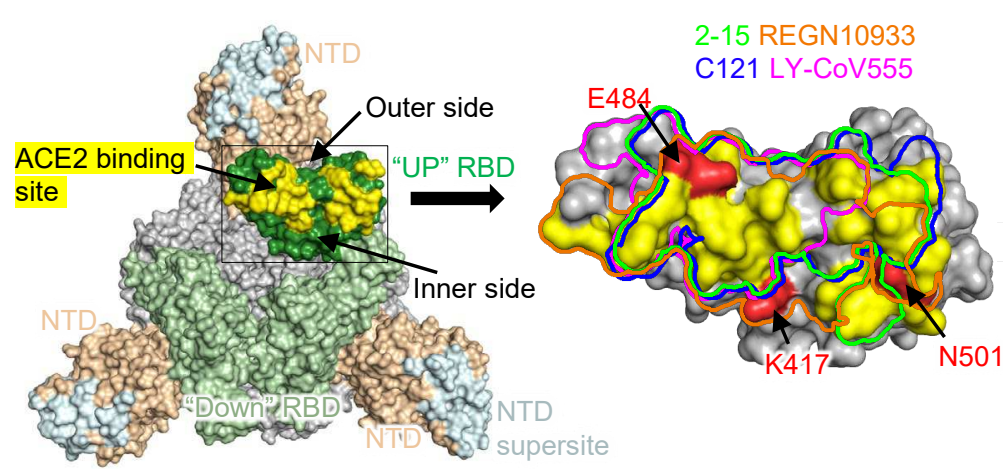

Top view of spike

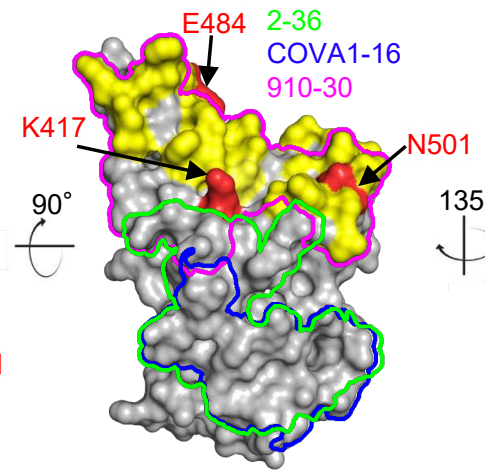

Inner side view

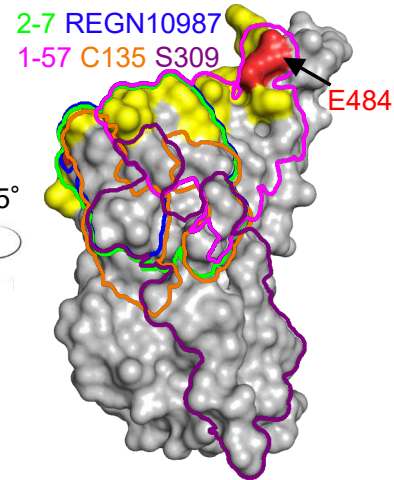

Outer side view

b

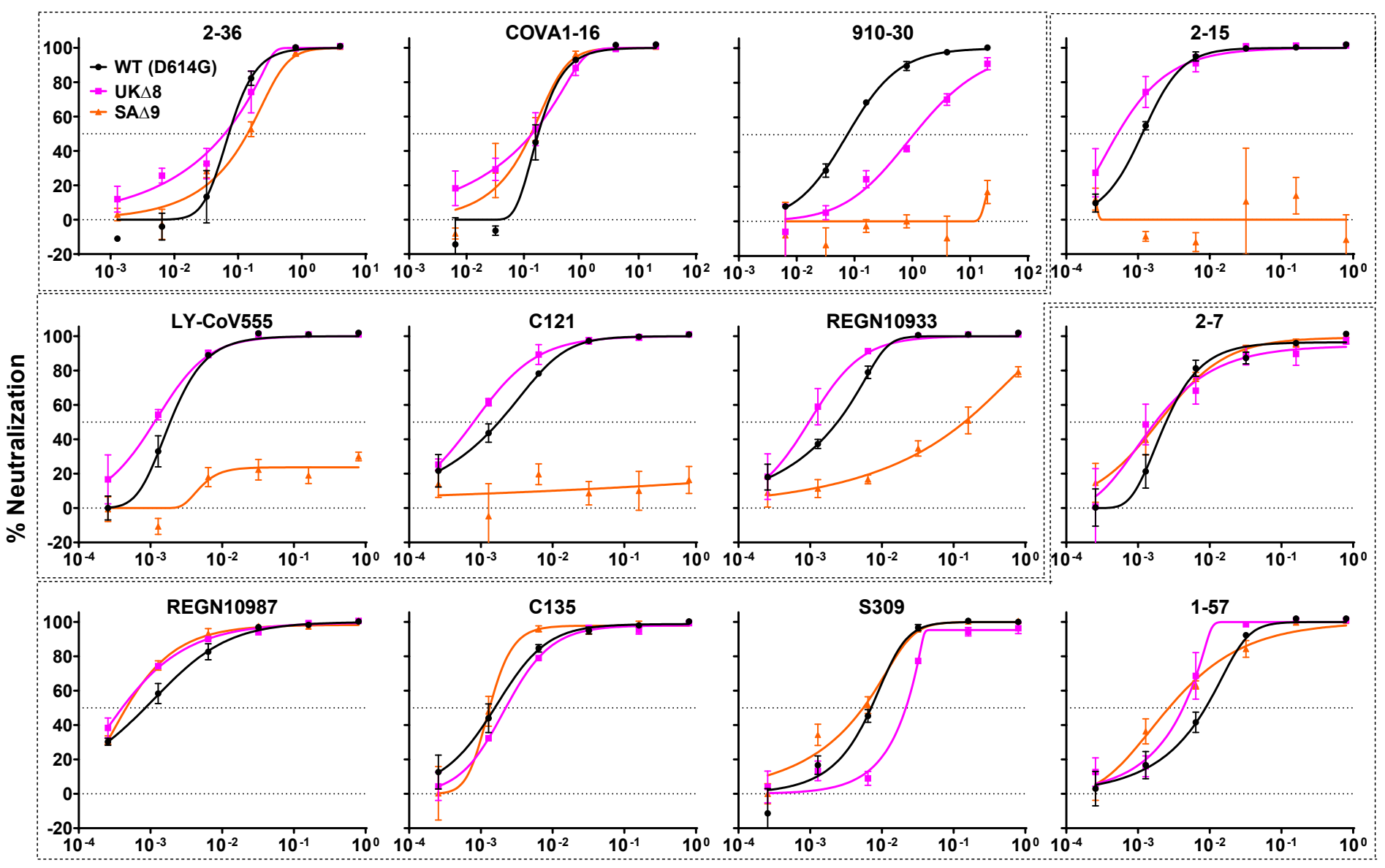

Antibody $(\mu \mathrm{g} / \mathrm{mL})$ 
Fig. 2

C

\begin{tabular}{|c|c|c|c|c|c|c|c|c|c|c|c|c|c|c|c|c|c|c|c|}
\hline \multirow{3}{*}{\multicolumn{2}{|c|}{$\begin{array}{l}\text { Fold Change of } \\
\text { IC50 from WT }\end{array}$}} & \multicolumn{12}{|c|}{ RBD-directed mAbs } & \multicolumn{6}{|c|}{ NTD-directed mAbs } \\
\hline & & \multicolumn{3}{|c|}{ Inner side } & \multicolumn{4}{|c|}{ RBM } & \multicolumn{5}{|c|}{ Outer side } & \multicolumn{4}{|c|}{ Supersite } & \multicolumn{2}{|c|}{ Others } \\
\hline & & $2-36$ & CovA1-16 & $910-30$ & $2-15$ & LY-CoV555 & C121 & REGN10933 & $2-7$ & REGN10987 & C135 & S309 & $1-57$ & $5-24$ & 4-8 & 4A8 & $2-17$ & 4-19 & $5-7$ \\
\hline \multirow{9}{*}{ UK } & UK $\Delta 8$ & 1.2 & 1.3 & -14.0 & 2.2 & 1.7 & 2.3 & 2.5 & 1.4 & 2.1 & -1.4 & -3.1 & 2.1 & $<-1000$ & $<-1000$ & $<-1000$ & -121.2 & -20.5 & 4.2 \\
\hline & 69-70del & -1.0 & 1.1 & 2.7 & 1.2 & 1.1 & 1.7 & 1.3 & -1.2 & 1.2 & 1.8 & -1.6 & 1.1 & 1.1 & 1.1 & 1.5 & -1.1 & -3.6 & 1.4 \\
\hline & 144del & 1.5 & -1.3 & 2.3 & 1.3 & 1.1 & 1.7 & 1.3 & 1.2 & -1.4 & 1.4 & 1.4 & 1.1 & $<-1000$ & $<-1000$ & $<-1000$ & -80.7 & 1.6 & 1.7 \\
\hline & N501Y & -1.2 & -1.4 & -12.7 & 1.5 & -1.0 & 1.5 & -1.4 & -1.0 & 1.3 & 1.2 & 1.2 & 3.6 & -2.9 & -6.7 & $\mathrm{MPI} \downarrow$ & -12.0 & -1.4 & 1.5 \\
\hline & A570D & 4.1 & 1.9 & 6.7 & 1.4 & 1.7 & 1.7 & 4.7 & -2.3 & $\begin{array}{l}-1.6 \\
\end{array}$ & 1.1 & -1.2 & 2.2 & 1.1 & -15.1 & -2.9 & -4.8 & -1.9 & 2.3 \\
\hline & $\mathrm{P} 681 \mathrm{H}$ & 2.0 & 1.5 & 2.5 & 3.1 & 2.3 & -1.0 & 1.6 & -1.4 & -1.9 & 1.3 & -1.2 & 2.9 & -1.5 & -2.8 & 1.1 & -4.7 & -1.2 & 1.1 \\
\hline & T716I & 4.3 & 3.9 & 3.9 & 3.1 & 3.5 & 2.0 & 3.6 & -1.1 & -1.6 & 1.2 & -1.6 & 2.9 & -3.5 & -5.5 & $\overline{\mathrm{MPI} \downarrow}$ & -2.6 & 1.2 & 1.8 \\
\hline & S982A & -3.9 & -3.0 & -2.4 & 1.1 & -2.0 & 1.4 & -2.3 & -2.2 & -1.2 & 1.6 & -1.0 & -1.5 & -1.1 & -1.1 & -2.9 & -4.3 & 1.2 & -1.3 \\
\hline & $\mathrm{D} 1118 \mathrm{H}$ & -1.1 & -3.1 & 1.0 & 1.2 & 1.0 & 1.7 & -1.3 & -1.4 & -1.7 & 1.2 & 1.5 & 1.1 & -1.3 & -3.1 & 1.4 & -1.1 & -1.1 & -1.0 \\
\hline & & & & & & & & & & & & & & & & & & & \\
\hline \multirow{10}{*}{ SA } & SA $\triangle 9$ & -2.0 & 1.3 & $<-1000$ & $<-1000$ & $\mid<-1000$ & $<-1000$ & -58.8 & 1.3 & 1.8 & 1.2 & 1.3 & 3.3 & $<-1000$ & $<-1000$ & $<-1000$ & -406.6 & $<-1000$ & 4.0 \\
\hline & L18F & 1.5 & 1.9 & 2.8 & 3.0 & 1.0 & 1.8 & 1.4 & -1.4 & -1.8 & 1.1 & 1.2 & -1.6 & -2.2 & 1.3 & $\mathrm{MPI} \downarrow$ & -107.2 & $<-1000$ & 1.4 \\
\hline & D80A & -1.4 & 1.2 & 2.1 & 2.0 & 1.5 & 2.0 & 1.4 & -2.2 & -2.2 & 1.0 & 2.2 & -2.7 & 2.3 & 2.0 & -1.0 & -2.0 & $<-1000$ & 2.2 \\
\hline & D215G & 1.9 & 1.6 & 1.5 & 1.8 & 1.5 & 2.1 & 1.5 & -1.8 & -2.1 & -1.2 & 1.0 & 2.2 & -1.1 & -1.8 & -2.3 & -6.0 & 1.1 & -1.2 \\
\hline & 242-244del & -1.4 & 1.2 & -1.2 & 1.4 & -1.1 & 1.1 & 1.0 & -1.2 & -3.2 & 1.8 & 1.2 & -1.3 & $<-1000$ & $<-1000$ & $<-1000$ & $<-1000$ & $<-1000$ & -1.3 \\
\hline & R246I & 1.3 & 1.7 & 2.2 & 2.4 & 1.4 & 2.1 & 2.2 & 1.4 & -2.1 & 1.1 & 2.3 & 1.7 & $<-1000$ & $<-1000$ & $<-1000$ & -2.8 & $<-1000$ & 3.1 \\
\hline & K417N & 3.2 & 3.3 & $<-1000$ & 3.3 & 8.4 & 1.2 & -13.1 & 2.1 & -1.2 & 2.9 & 1.6 & 7.8 & 2.9 & -1.6 & 1.7 & -1.5 & 1.2 & 5.0 \\
\hline & $\mathrm{E} 484 \mathrm{~K}$ & -1.2 & -1.0 & 4.3 & $<-1000$ & $<-1000$ & $<-1000$ & -10.5 & -3.4 & -1.1 & 2.3 & 2.5 & -1.1 & -1.6 & -3.2 & $\mathrm{MPI} \downarrow$ & -2.8 & -1.1 & -3.9 \\
\hline & N501Y & -1.2 & -1.4 & -12.7 & 1.5 & -1.0 & 1.5 & -1.4 & -1.0 & 1.3 & 1.2 & 1.2 & 3.6 & -2.9 & -6.7 & $\overline{M P I \downarrow}$ & -12.0 & -1.4 & 1.5 \\
\hline & A701V & 1.9 & 1.4 & 2.1 & 2.8 & 2.0 & 1.6 & 2.3 & -1.8 & -2.6 & 1.5 & 1.1 & 2.5 & -3.3 & -2.0 & $\mathrm{MPI}$ & -3.3 & -1.2 & -1.0 \\
\hline
\end{tabular}

Red: resistance $>3$ fold; Green: sensitization $>3$ fold

d

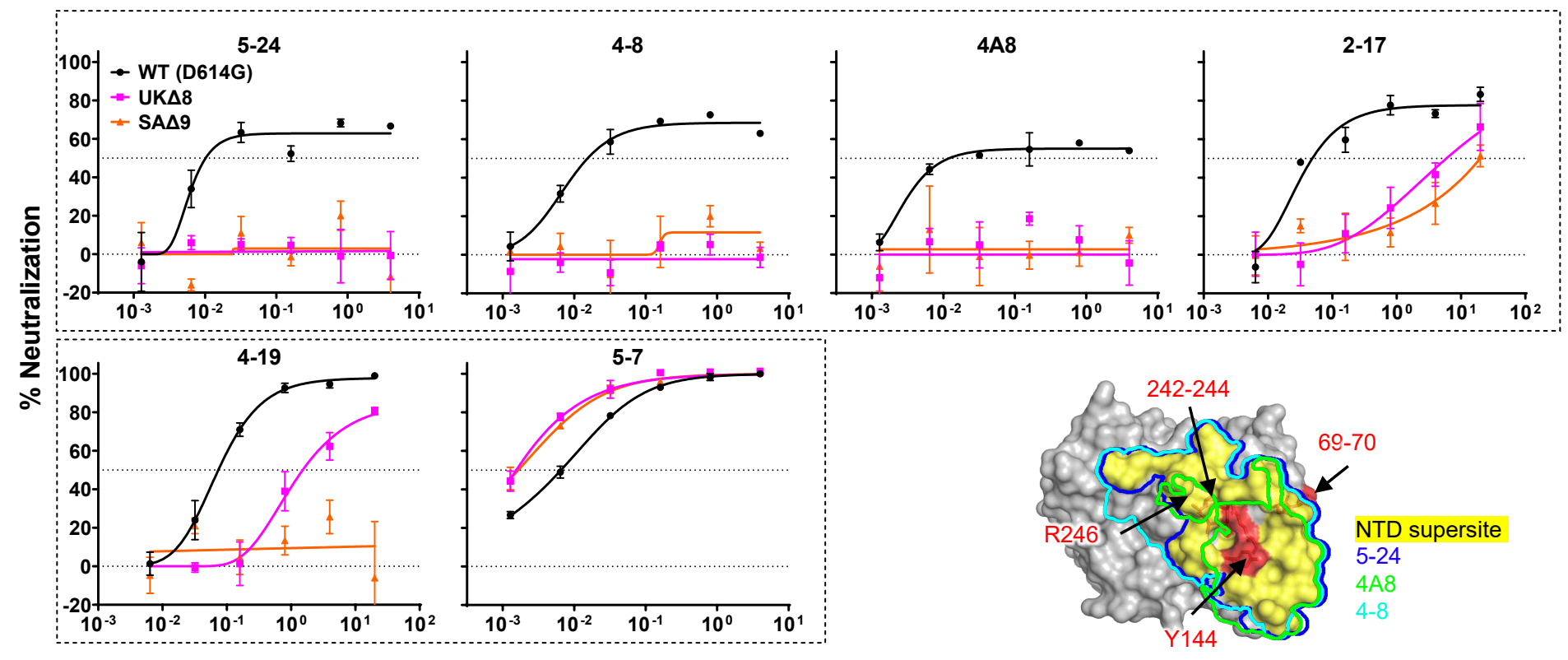

Antibody $(\mu \mathrm{g} / \mathrm{mL})$ 
Fig. 2

e

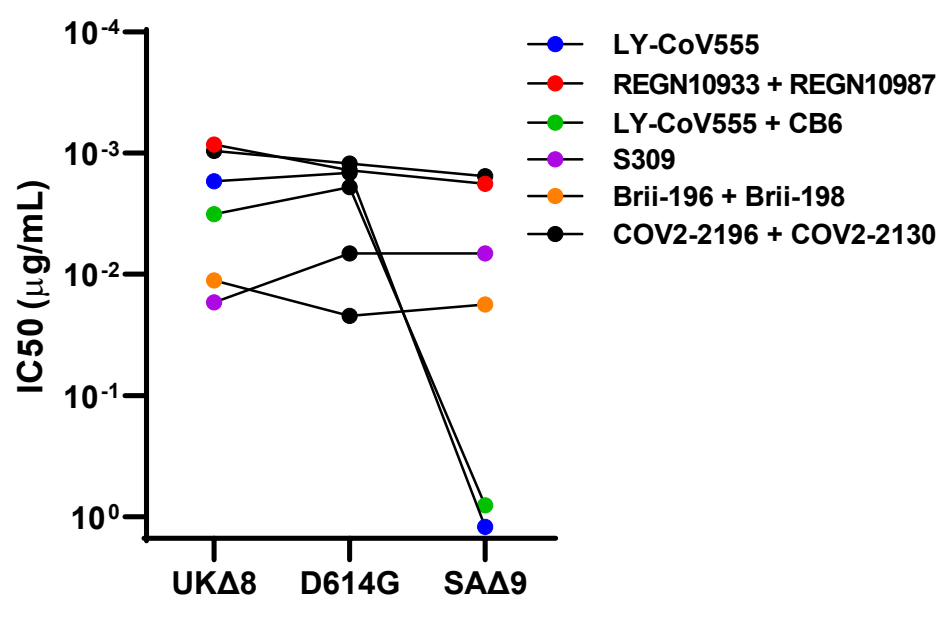


Fig. 3
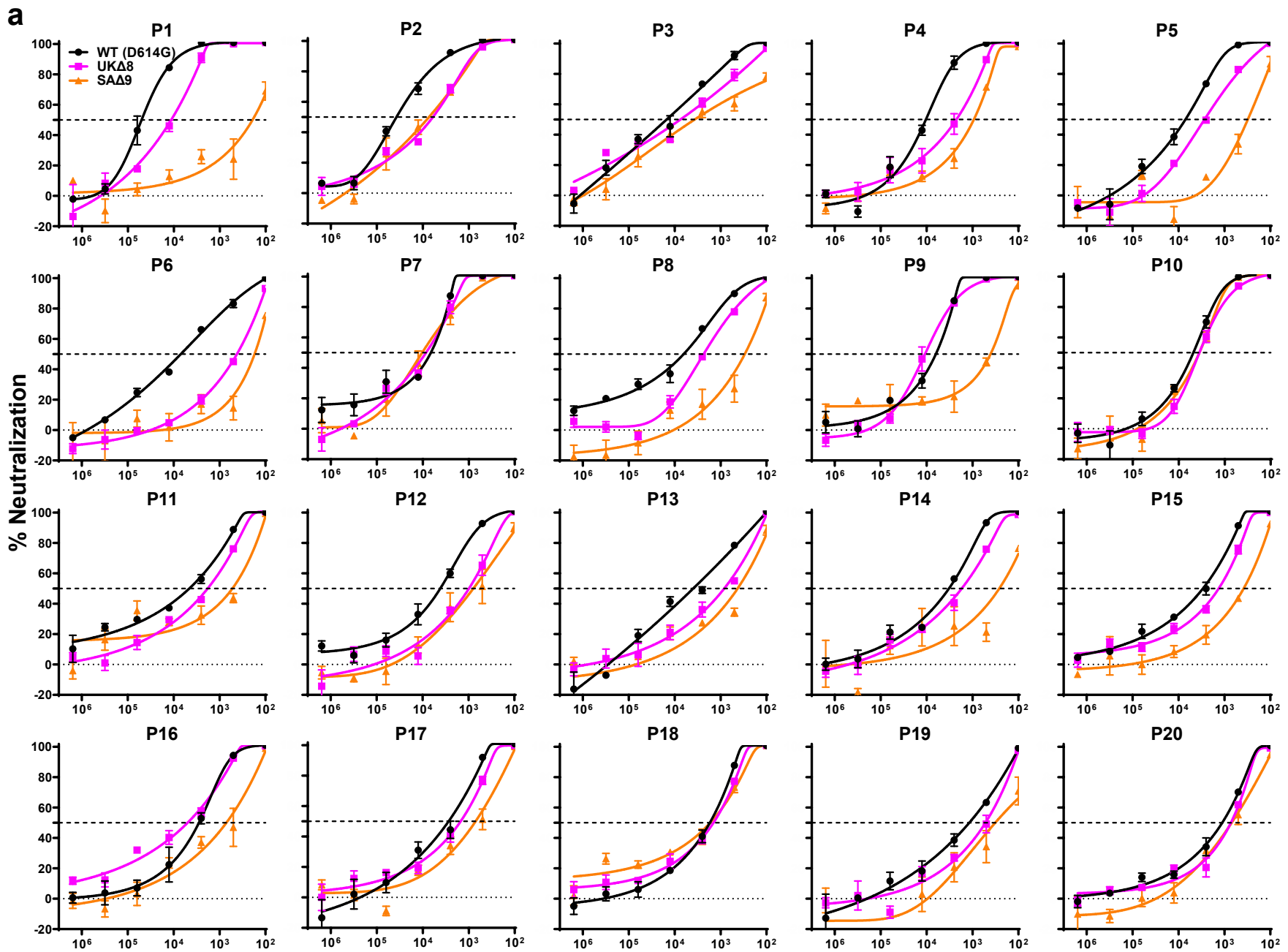

Reciprocal serum dilution

b

\begin{tabular}{|c|c|c|c|c|c|c|c|c|c|c|c|c|c|c|c|c|c|c|c|c|c|}
\hline \multirow{2}{*}{\multicolumn{2}{|c|}{$\begin{array}{l}\text { Fold change of } \\
\text { IC50 from WT }\end{array}$}} & \multicolumn{20}{|c|}{ Convalescent plasma } \\
\hline & & P1 & P2 & P3 & P4 & P5 & P6 & $\mathbf{P 7}$ & P8 & P9 & $\mathbf{P 1 0}$ & P11 & $\mathbf{P 1 2}$ & P13 & P14 & P15 & P16 & P17 & P18 & P19 & P20 \\
\hline \multirow{6}{*}{ UK } & UK $\Delta 8$ & -4.4 & -6.2 & -2.0 & -4.6 & -2.6 & -16.7 & +1.3 & -2.7 & +1.7 & -1.4 & -2.5 & -4.2 & -4.7 & -1.9 & -2.2 & +1.8 & -1.8 & -1.2 & -2.3 & -1.5 \\
\hline & 144del & +1.3 & +2.8 & +1.4 & +2.6 & -1.4 & -4.5 & -1.1 & -1.5 & 1.0 & -1.1 & -4.5 & 1.1 & -2.2 & -1.4 & +1.1 & -1.6 & -2.0 & -1.5 & 2.0 & -1.4 \\
\hline & N501Y & -1.6 & -2.3 & +1.9 & 1.0 & -1.1 & -3.6 & 1.0 & -2.4 & +1.5 & +1.2 & -2.0 & -2.1 & -3.1 & -1.3 & -1.7 & -1.3 & -1.5 & 1.0 & -1.3 & +1.4 \\
\hline & A570D & 1.0 & +4.3 & +1.9 & +5.1 & -1.1 & -3.2 & +1.4 & -1.6 & +1.5 & +1.4 & -2.7 & +1.4 & -3.1 & +1.1 & -1.1 & -1.1 & -1.2 & -1.1 & -1.0 & -1.0 \\
\hline & S982A & -5.0 & -9.3 & +1.2 & -1.5 & -2.5 & -2.8 & 1.0 & -3.0 & $\begin{array}{r}+1.2 \\
\end{array}$ & +1.1 & -2.2 & -2.7 & -3.7 & -1.4 & -1.4 & -1.1 & -2.0 & +1.2 & -2.4 & -1.7 \\
\hline & $\mathrm{D} 1118 \mathrm{H}$ & -2.1 & -1.9 & +2.0 & +1.1 & -1.5 & -2.6 & +1.0 & -3.1 & +1.2 & -1.1 & -2.6 & -1.4 & -3.0 & 1.0 & -1.7 & -1.3 & -1.7 & -1.1 & -1.5 & 1.0 \\
\hline \multirow{6}{*}{ SA } & SA $\Delta 9$ & 260.6 & -5.1 & -4.1 & -11.1 & -22.8 & -40.4 & +1.6 & -21.4 & -15.5 & -1.4 & -8.7 & -5.2 & -9.3 & -12.5 & -7.7 & -4.0 & -3.9 & 1.0 & -3.7 & -1.6 \\
\hline & L18F & -1.2 & 1.0 & +1.9 & +3.0 & -1.9 & +1.7 & +1.5 & -1.1 & +1.5 & +1.1 & +1.9 & -1.1 & -1.5 & +1.3 & -1.2 & +2.1 & +1.3 & -1.1 & +1.8 & 1.0 \\
\hline & $\mathrm{K} 417 \mathrm{~N}$ & -1.3 & 1.4 & +6.6 & +2.5 & -1.1 & -2.0 & +1.8 & 1.0 & +1.8 & +1.2 & -1.6 & -1.4 & -2.1 & +1.8 & -1.2 & +1.3 & -1.1 & 1.2 & -1.2 & +1.5 \\
\hline & E484K & -25.4 & -4.7 & -1.3 & -2.6 & -7.6 & -9.6 & -1.6 & -10.8 & -9.1 & -1.3 & -8.1 & -3.5 & -9.8 & -2.3 & -6.3 & -4.3 & -3.3 & -1.5 & -4.0 & -3.5 \\
\hline & N501Y & -1.6 & -2.3 & +1.9 & 1.0 & -1.1 & -3.6 & 1.0 & -2.4 & +1.5 & +1.2 & -2.0 & -2.1 & -3.1 & -1.3 & -1.7 & -1.3 & -1.5 & -1.0 & -1.3 & +1.4 \\
\hline & A701V & -1.3 & -3.8 & -1.1 & -1.2 & -1.9 & -2.3 & -1.0 & -2.1 & +1.4 & +1.1 & -2.9 & -1.5 & -2.3 & -1.1 & -1.8 & -1.7 & -1.7 & -1.7 & -1.9 & -1.1 \\
\hline
\end{tabular}

Red: resistance $>2$ fold; Green: sensitization $>2$ fold 
Fig. 3

C

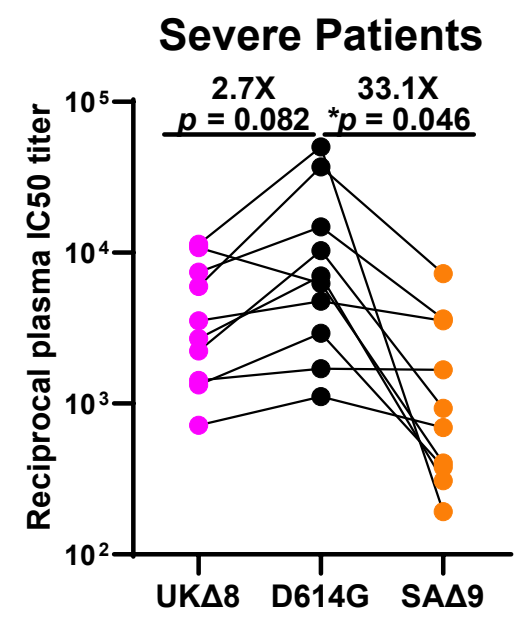

\section{Non-severe Patients}

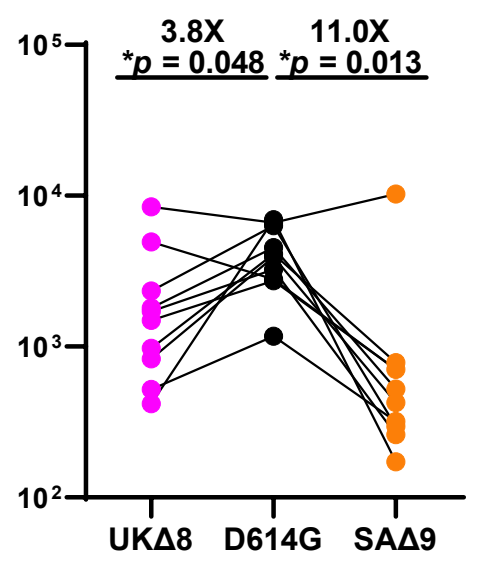


Fig. 4

a
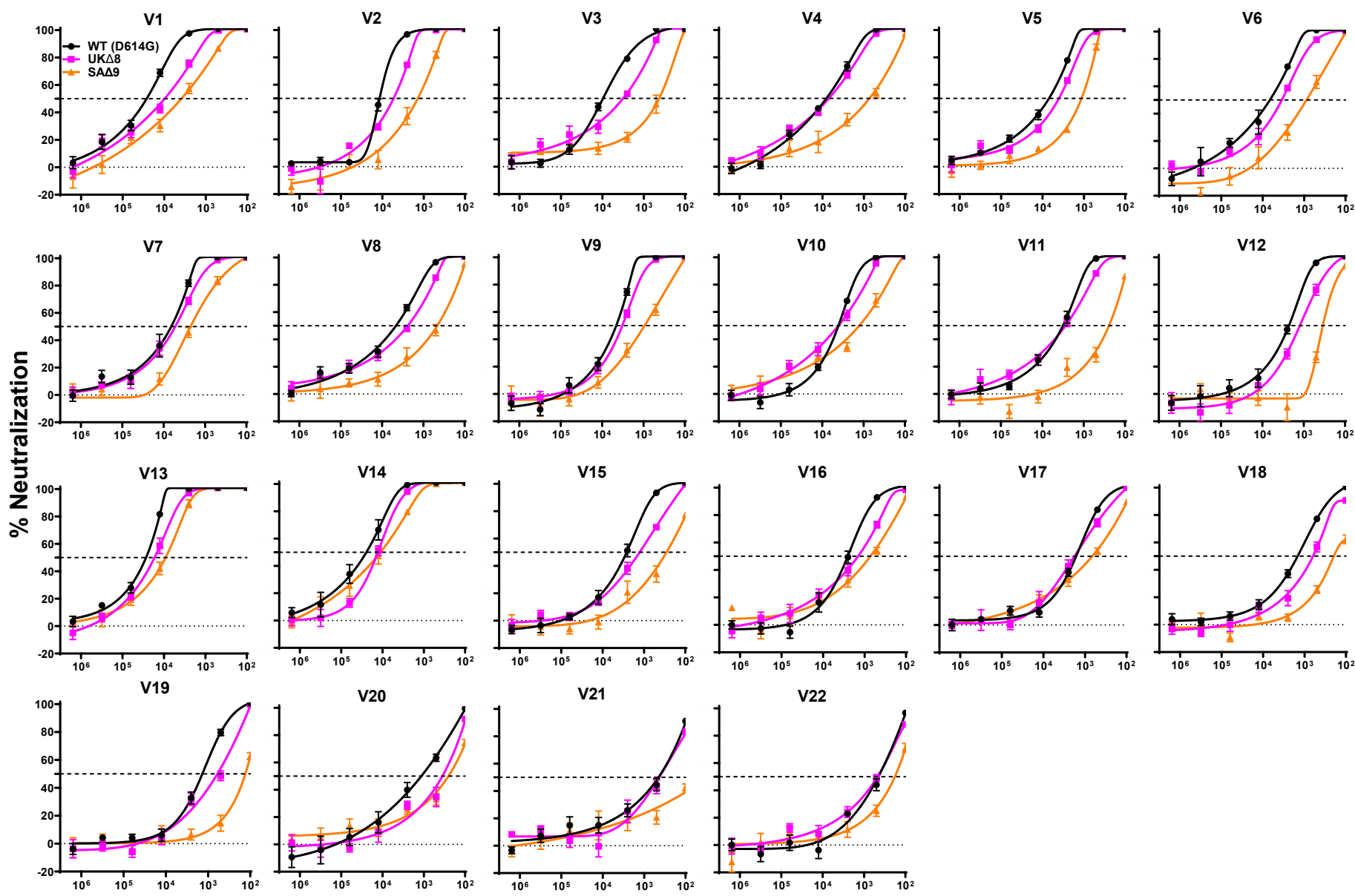

Reciprocal serum dilution

b

\begin{tabular}{|c|c|c|c|c|c|c|c|c|c|c|c|c|c|c|c|c|c|c|c|c|c|c|c|}
\hline \multirow{2}{*}{\multicolumn{2}{|c|}{$\begin{array}{l}\text { Fold change of } \\
\text { IC50 from WT }\end{array}$}} & \multicolumn{12}{|c|}{ Moderna vaccinee sera } & \multicolumn{10}{|c|}{ Pfizer vaccinee sera } \\
\hline & & V1 & V2 & V3 & V4 & V5 & V6 & V7 & V8 & V9 & V10 & V11 & V12 & V13 & V1 & V15 & V16 & V17 & V18 & V19 & V20 & V21 & V22 \\
\hline \multirow{9}{*}{ UK } & UK $\Delta 8$ & -2.7 & -2.2 & -3.0 & -1.2 & -1.7 & -1.9 & -1.2 & -1.9 & -1.4 & 1.0 & -1.2 & -1.8 & -1.6 & $-1 . c$ & -2.1 & -1.8 & +1.1 & -2.4 & -2.3 & -3.2 & -1.1 & +1.1 \\
\hline & 69-70del & +1.4 & +1.4 & \begin{tabular}{|l|}
-1.3 \\
\end{tabular} & +1.2 & $\begin{array}{ll}-1.3 \\
\end{array}$ & -1.1 & +1.9 & +1.1 & +1.5 & -1.4 & +1.3 & +1.3 & -1.4 & +1. & -1.0 & -1.0 & -1.5 & -1.3 & +1.3 & +1.3 & -1.4 & +2.1 \\
\hline & 144del & -1.1 & -1.2 & -1.4 & +2.1 & -1.2 & -1.2 & -1.1 & -1.2 & -1.2 & -1.1 & +1.1 & -1.3 & -1.2 & -1.7 & -1.2 & -1.2 & -1.3 & -1.1 & -1.2 & -1.6 & +1.1 & -1.3 \\
\hline & N501Y & +1.5 & +1.1 & -1.8 & +1.6 & -2.0 & +1.9 & +2.2 & -2.0 & -1.2 & +4.6 & +2.9 & -1.2 & -1.2 & +1.2 & -2.1 & -1.6 & -1.6 & -1.5 & -1.1 & -2.4 & -1.4 & 1.0 \\
\hline & A570D & +1.4 & +2.2 & +1.2 & +2.4 & +1.7 & +1.6 & +2.2 & +1.5 & 1.0 & +1.5 & +1.4 & +1.6 & +1.2 & +1 & +1.5 & +2 & +1.2 & +1.3 & +1.8 & +1.1 & -1.2 & +1.4 \\
\hline & $\mathrm{P} 681 \mathrm{H}$ & +2.2 & +1.2 & -1.7 & -1.5 & -1.5 & +1.0 & +1.1 & -1.4 & 1.0 & -1.1 & +1.1 & +1.1 & +1.2 & +1.2 & -1.3 & +1 & -1.1 & -1.1 & \begin{tabular}{|l|}
-1.0 \\
\end{tabular} & -2.1 & -1.3 & -1.0 \\
\hline & T716I & +1.1 & -1.1 & -1.1 & +1.6 & +1.3 & +1.3 & +1.8 & +1.1 & 1.0 & +1.6 & +1.2 & +1.4 & +1.7 & +1. & +1.3 & +1 & +1.1 & +1.3 & +1.1 & +1.6 & +1.1 & +1.1 \\
\hline & S982A & -2.3 & -1.5 & -2.6 & -1.8 & -2.0 & -1.6 & -1.3 & -2.5 & -1.7 & -1.5 & -1.5 & -1.5 & -1.2 & -1.6 & -1.6 & -1.7 & -1.5 & -1.9 & \begin{tabular}{|l|}
-1.6 \\
\end{tabular} & -2.4 & -2.0 & -1.3 \\
\hline & $\mathrm{D} 1118 \mathrm{H}$ & -1.2 & +1.1 & -1.2 & -1.4 & +1.1 & 1.0 & 1.0 & -1.5 & -1.2 & -1.1 & 1.0 & +1.1 & -1.2 & -1.2 & -1.6 & -1.6 & -1.2 & -1.5 & -1.2 & -1.3 & -1.7 & -1.3 \\
\hline \multirow{10}{*}{ SA } & SA $\Delta 9$ & -6.9 & -8.4 & -22.7 & -11.0 & -6.1 & -7.7 & -2.9 & -10.0 & -4.8 & -3.2 & -13.0 & -6.6 & -3.0 & -2.2 & -9.2 & -3.5 & -2.5 & -7.5 & -10.4 & -4.5 & -15.0 & -2.5 \\
\hline & L18F & +1.9 & 1.0 & -1.8 & -1.1 & -1.3 & 1.0 & +3.3 & -1.5 & 1.0 & +1.2 & +1.8 & 1.0 & +1.2 & -1.3 & +1.2 & +1.2 & 1.0 & -1.4 & +1.4 & -1.4 & -1.5 & +1.4 \\
\hline & D80A & +1.2 & +1.5 & -1.1 & +2.1 & +1.1 & +1.5 & +1.8 & -1.5 & +1.4 & +3.0 & +1.3 & +1.1 & +1.8 & 1.0 & +1.2 & +1 & -1.5 & -1.8 & 1.0 & -1.8 & -1.3 & +1.1 \\
\hline & D215G & -1.3 & +1.1 & +1.1 & -1.2 & +1.3 & +1.2 & -1.1 & -2.9 & -1.1 & +2.7 & +1.1 & -1.3 & -1.1 & +1 & -1.8 & -2. & -1.2 & -1.8 & -1.3 & 1.0 & -1.2 & +1.1 \\
\hline & 242-244del & -3.6 & 1.0 & \begin{tabular}{|l|}
-1.3 \\
\end{tabular} & -1.8 & -1.7 & -1.3 & -1.7 & -1.7 & -1.4 & -1.5 & -1.6 & -1.6 & -1.4 & -1.9 & -1.4 & -1 & +1.5 & +1.1 & \begin{tabular}{|l|}
-1.3 \\
\end{tabular} & -2.6 & -1.6 & -1.8 \\
\hline & R246I & -1.6 & +1.1 & -2.0 & -1.1 & -1.7 & -1.2 & +1.1 & -2.1 & -1.3 & -1.1 & 1.0 & -1.7 & -1.5 & -1.2 & +1.6 & 1.0 & +1.3 & +2.0 & +2.9 & -4.0 & 1.0 & -1.1 \\
\hline & K417N & +1.6 & +1.4 & -1.1 & 1.0 & 1.0 & -1.2 & +1.7 & -1.3 & +1.1 & +1.4 & -1.3 & +1.5 & +1.4 & +1.8 & +1.2 & +1 & -1.5 & +1.9 & +2.0 & 1.0 & -1.8 & +1.6 \\
\hline & E484K & -3.0 & -2.3 & -3.9 & -4.0 & -1.4 & -2.8 & -1.3 & -3.3 & -2.2 & -2.6 & -3.2 & -1.8 & -1.9 & -2.7 & -2.1 & -1. & $-2 . c$ & -11.3 & -3.3 & -3.2 & -3.1 & -1.8 \\
\hline & N501Y & +1.5 & +1.1 & -1.8 & +1.6 & -2.0 & +1.9 & +2.2 & -2.0 & -1.2 & +4.6 & +2.9 & -1.2 & -1.2 & +1.2 & -2.1 & -1. & -1.6 & -1.5 & \begin{tabular}{|l|}
-1.1 \\
\end{tabular} & -2.4 & -1.4 & 1.0 \\
\hline & A701V & -1.1 & -1.2 & -1.9 & -2.2 & -1.7 & -1.6 & -1.4 & -1.7 & +1.2 & +1.1 & +2.1 & -1.1 & -1.2 & -1.3 & \begin{tabular}{|l|}
-1.2 \\
\end{tabular} & -1. & -2. & -1.5 & \begin{tabular}{|l|}
-1.4 \\
\end{tabular} & +1.1 & -2.2 & -1.5 \\
\hline
\end{tabular}

Red: resistance $>2$ fold; Green: sensitization $>2$ fold 
Fig. 4

C

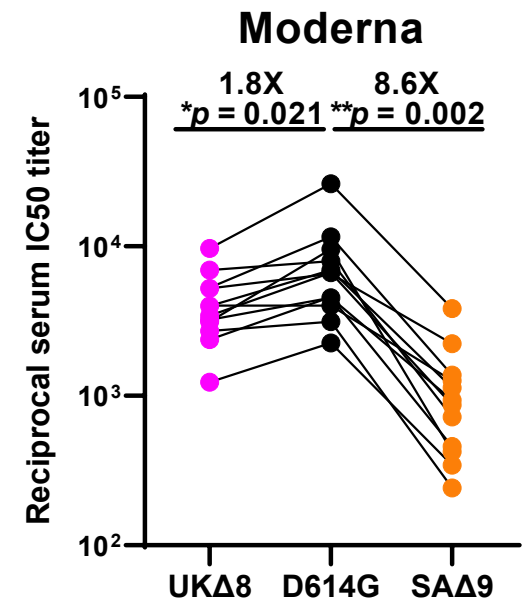

Pfizer

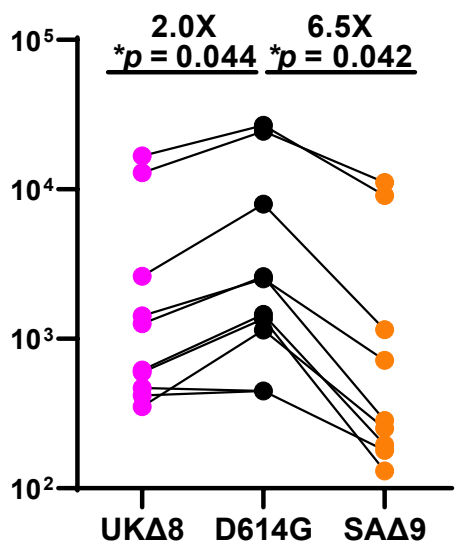




\section{Figures}

a

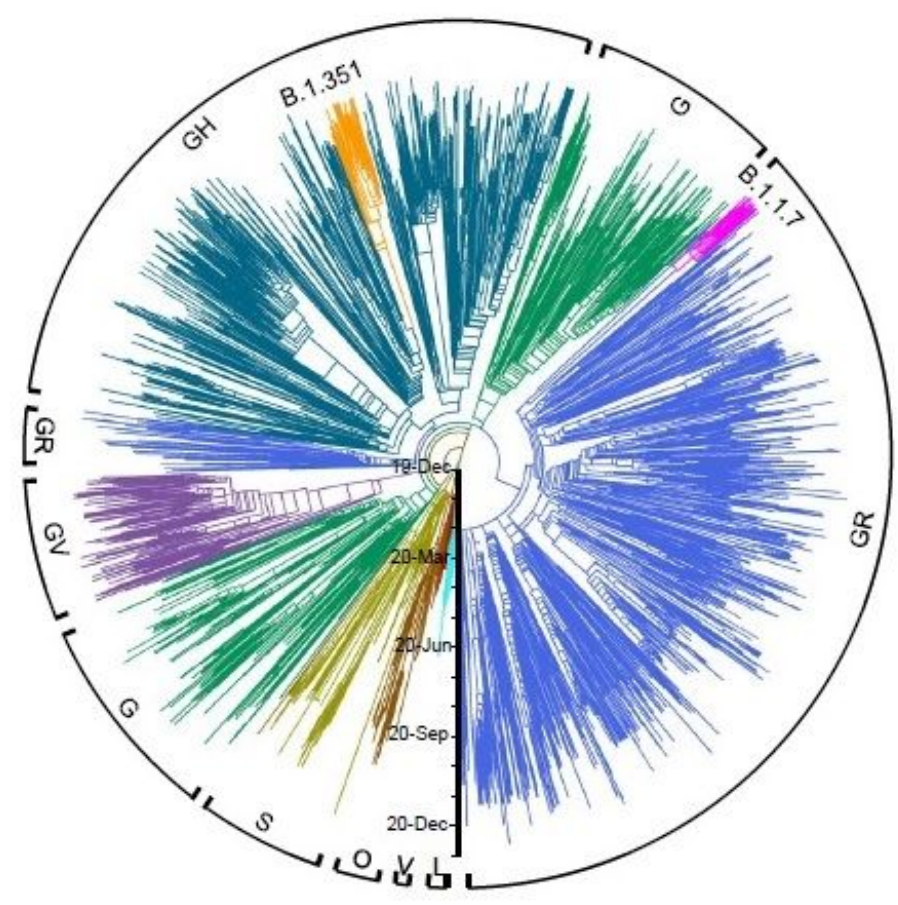

b

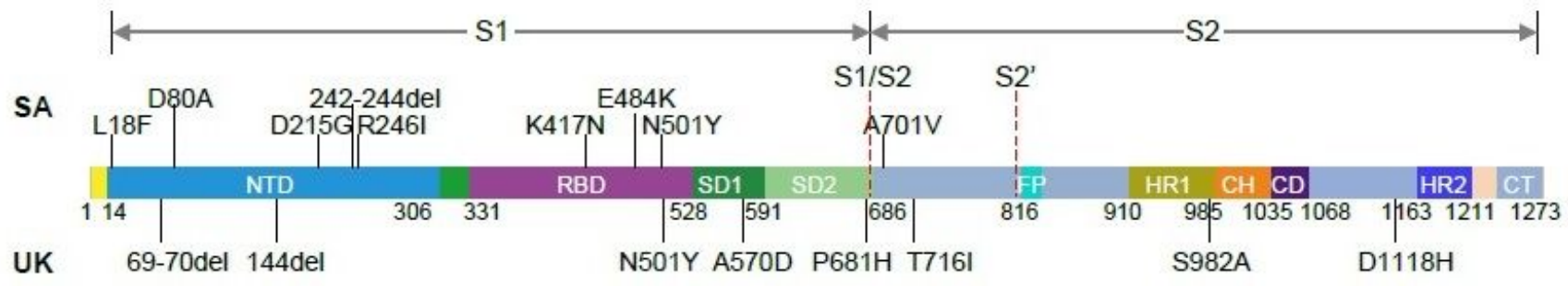

Figure 1

Emerging SARS-CoV-2 variants identified in the UK and SA. a, Phylogenetic tree of SARS-CoV-2 variants, with B.1.351 and B.1.1.7 highlighted. b, Mutations in the viral spike identified in B.1.351 (SA) and B.1.1.7 (UK) in addition to D614G. 


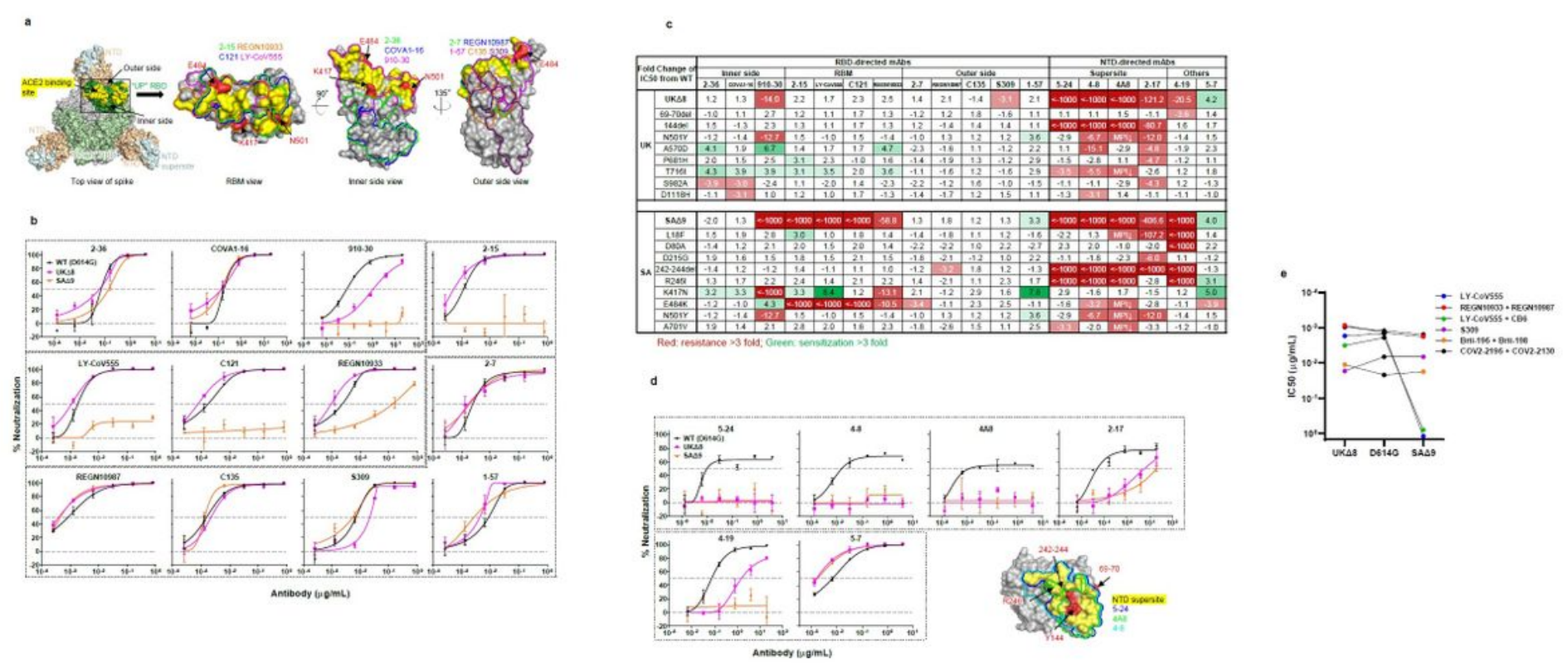

Figure 2

Susceptibility of UK $\triangle 8$ and SA $\Delta 9$ pseudoviruses to neutralization by mAbs. a, Footprints of neutralizing mAbs on the RBD. Left panel, top view of SARS-COV-2 spike with one RBD in the "up" conformation (pdb: $6 z g g)$. RBD and NTD are colored green and peach, respectively. The positions of 'inner' and 'outer' sides are indicated on the "up" RBD with the ACE2-binding site colored yellow. The three panels to the right show the antibody footprints on RBD. b, Neutralization of UK $\triangle 8, \mathrm{SA} \triangle 9$, and WT pseudoviruses by select RBD mAbs. c, Fold-change in IC50 of neutralizing mAbs against UK $\Delta 8$ and $\mathrm{SA} \Delta 9$, as well as singlemutation pseudoviruses, relative to WT. MPI $\triangle$ denotes that maximum percent inhibition is substantially reduced, confounding IC50 calculations. d, Neutralization of UK $\Delta 8$, SA $\Delta 9$, and WT pseudoviruses by NTDdirected $\mathrm{mAbs}$, the footprints of which are delineated by the color tracings in the insert. e, Changes in neutralization IC50 of authorized or investigational therapeutic mAbs against UK $\Delta 8$ and $S A \triangle 9$. Data in $b$ and $d$ are mean \pm SEM of technical triplicates, and represent one of two independent experiments. 


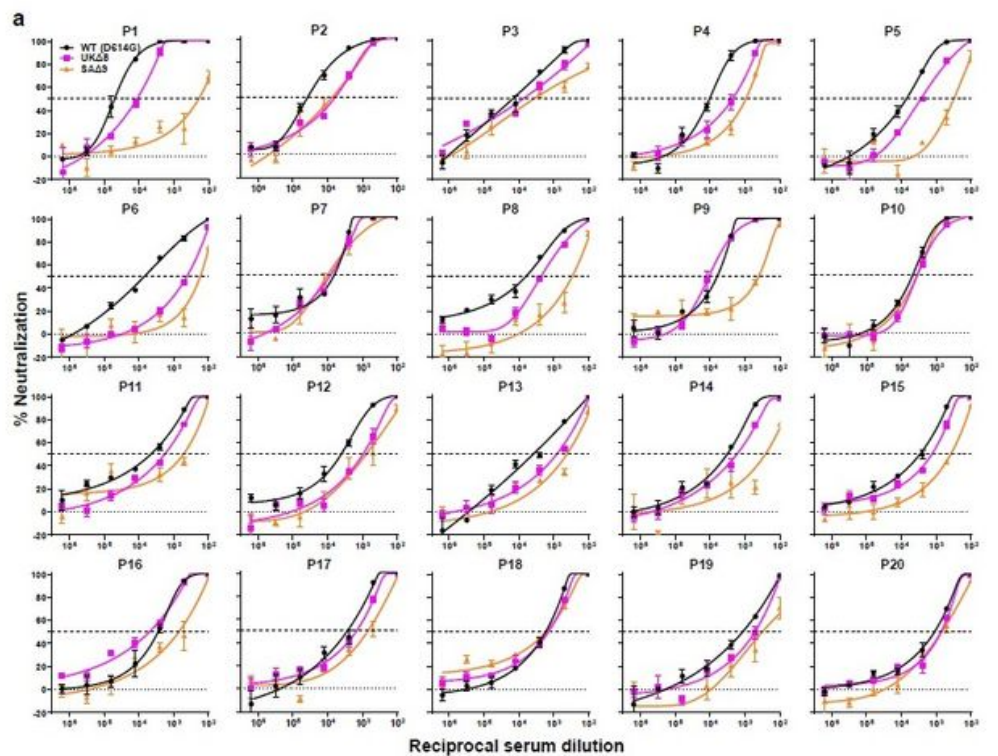

b

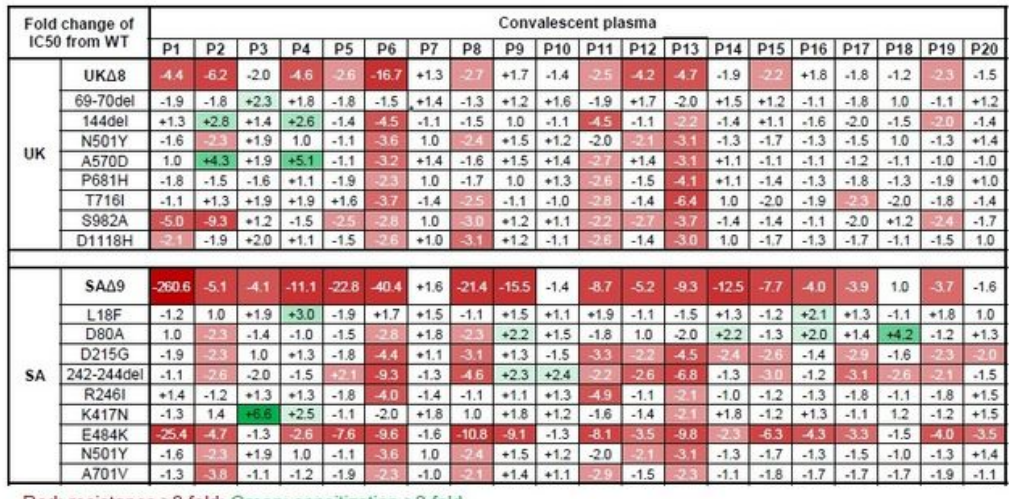

Red: resistance $>2$ fold; Green: sensitization $>2$ fold
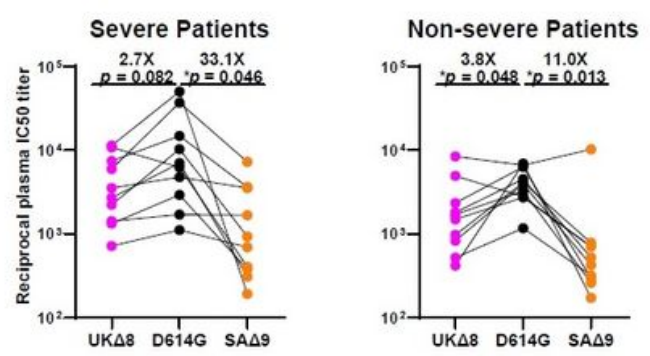

\section{Figure 3}

UK $\triangle 8$ and $\mathrm{SA} \triangle 9$ pseudoviruses are more resistant to neutralization by convalescent plasma from patients. a, Neutralization results for 20 convalescent plasma samples (P1-P20) against UK $\triangle 8, S A \Delta 9$, and WT. Data represent mean \pm SEM of technical triplicates. The panels are arranged by IC50 values against the WT, from low to high. b, Fold change in neutralization IC50 of UK $\Delta 8$ and SA $\Delta 9$, as well as single-mutation pseudoviruses, relative to the WT presented as a heatmap with darker colors implying greater change. c, Change in reciprocal plasma neutralization IC50 values of convalescent plasma from severe and non-severe patients against UK $\triangle 8$ and SA $\triangle 9$, relative to the WT. Mean fold changes in IC50 values relative to the WT are written above the $p$ values. Statistical analysis was performed using a twotailed paired $t$ test. 

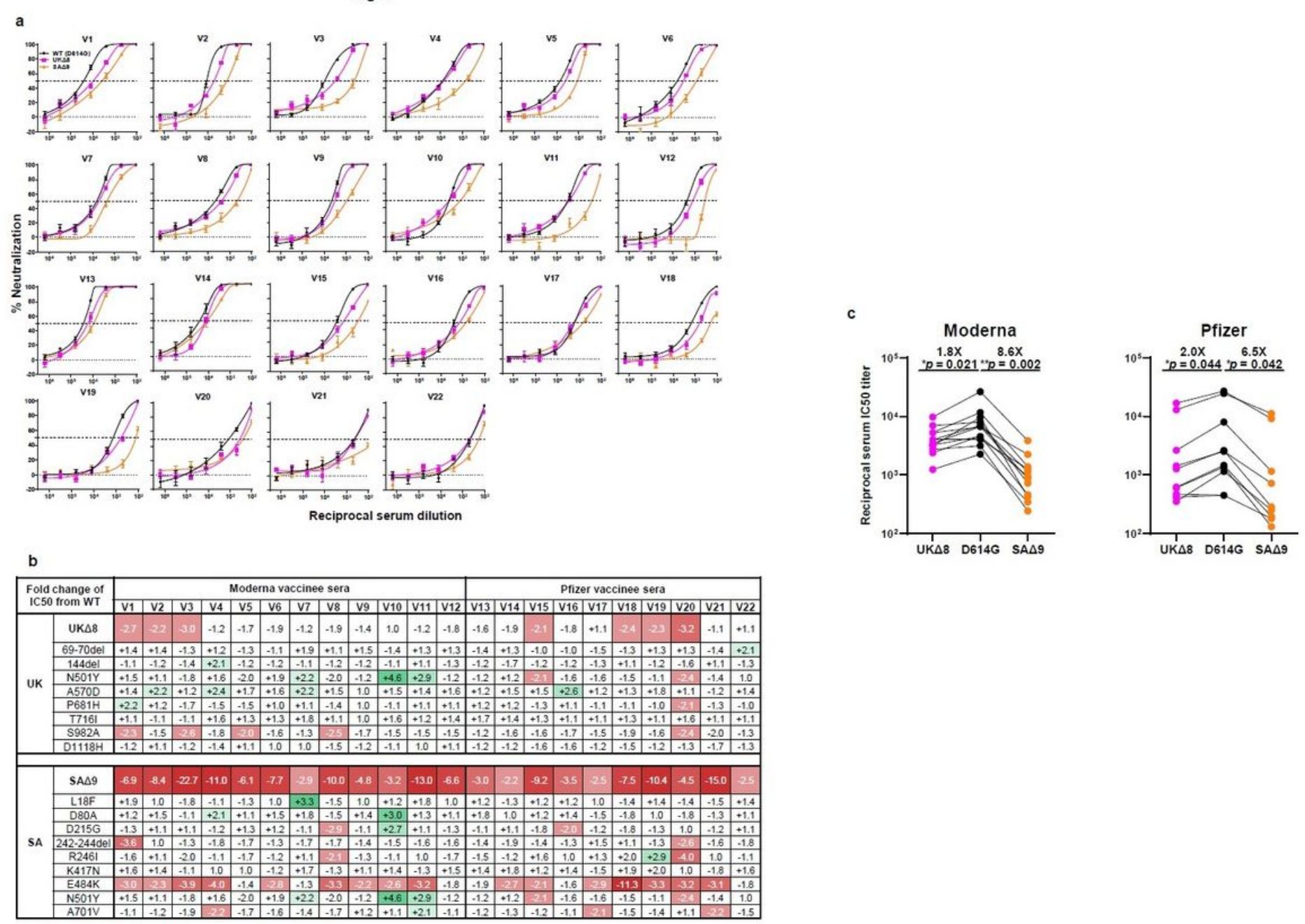

Red: resistance $>2$ fold; Green: sensitization $>2$ fold

\section{Figure 4}

UK $\triangle 8$ and $\mathrm{SA} \Delta 9$ pseudoviruses are more resistant to neutralization by vaccinee sera. a, Neutralization profiles for 22 serum samples obtained from persons who received SARS-CoV-2 vaccine made by Moderna (V1-V12) or Pfizer (V13-V22) against UK $\Delta 8, \mathrm{SA} \Delta 9$, and WT pseudoviruses. The panels are arranged by IC50 values against the WT, from low to high for each set of vaccinees. Data are mean \pm SEM of technical triplicates, and represent one of two independent experiments. $b$, Fold change in serum neutralization IC50 of UK $\triangle 8$ and $S A \Delta 9$, as well as single-mutation pseudoviruses, relative to the WT presented as a heatmap with darker colors implying greater change. c, Change in reciprocal serum IC50 values for Moderna and Pfizer vaccinees against UK $\Delta 8$ and $\mathrm{SA} \Delta 9$, relative to the WT. Mean fold change in IC50 relative to the WT is written above the $\mathrm{p}$ values. Statistical analysis was performed using a twotailed paired $t$ test.

\section{Supplementary Files}

This is a list of supplementary files associated with this preprint. Click to download. 
- Wang.et.al.Nature.1.25.supplemental.pdf 